\title{
Frustranes Weaning: Nachsorge durch das Weaningzentrum - Hausbesuche bei invasiv beatmeten Patienten
}

\author{
Weaning Failure: Follow-Up Care by the Weaning Centres - Home Visits \\ to Patients with Invasive Ventilation
}

Autoren

Institut
S. Stieglitz, W. Randerath

Krankenhaus Bethanien, Klinik für Pneumologie und Allergologie, Zentrum für Beatmungs- und Schlafmedizin, Solingen

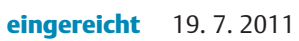
akzeptiert nach Revision 28. 9. 2011

\section{Bibliografie}

Dol http://dx.doi.org/ 10.1055/s-0031-1291439 Online-Publikation: 23.11 .2011 Pneumologie 2012; 66: 39-43 (c) Georg Thieme Verlag KG Stuttgart $\cdot$ New York ISSN 0934-8387

Korrespondenzadresse Dr. med. Sven Stieglitz Krankenhaus Bethanien Solingen

Aufderhöher Straße 169-175 42699 Solingen Sven.Stieglitz@klinik-bethanien. de

\section{Zusammenfassung \\ $\nabla$}

Hintergrund: Für die Betreuung außerklinisch invasiv beatmeter Patienten stehen derzeit keine flächendeckenden ärztlichen Versorgungsstrukturen zur Verfügung, so dass die medizinische Versorgung in aller erster Linie durch Pflegekräfte entsprechender Intensivpflegedienste erfolgt. Dabei wird sowohl in der Fachliteratur als auch in der aktuellen S2-Leitlinie „Nichtinvasive und invasive Beatmung als Therapie der chronischen respiratorischen Insuffizienz" für die Organisation der außerklinischen Beatmung eine poststationäre Mitbetreuung und eine Anbindung an das entlassende Beatmungszentrum empfohlen. Die Ausgestaltung der poststationären Mitbetreuung ist dabei offen gehalten.

Material und Methodik: Anhand von 6 Fällen werden exemplarisch die Gründe für die Notwendigkeit der Weiterbetreuung außerklinisch beatmeter Patienten durch das entlassende Weaningzentrum erörtert und erste Erfahrungen mit einem wöchentlichen Hausbesuch bei außerklinisch beatmeten Patienten berichtet.

Ergebnisse und Schlussfolgerung: Die Erfahrungen zeigen, dass es notwendig und praktikabel ist, bei diesen komplexen Fällen regelmäßige pneumologische Konsultationen in Form von Hausbesuchen durchzuführen. Werden die Daten aus der Betreuung von beatmeten Heimbewohnern zugrunde gelegt, ist zusätzlich von einer deutlichen Kostenersparnis im Gesundheitswesen auszugehen. Dennoch fehlen prospektive Studien, um den finanziellen und medizinischen Vorteil durch diese Versorgungsstruktur objektiv einschätzen zu können. Da das Patientengut mit außerklinischer Beatmung insgesamt sehr heterogen und die außerklinische Beatmung insgesamt selten ist, werden solche Studien multizentrisch ausgelegt sein müssen.

\section{Abstract \\ $\nabla$}

Backround: At present health-care at home for the majority of patients with invasive ventilation is mainly provided by nursing personnel. The reason for this unsatisfying condition is the lack of a basic medical supply for patients with invasive ventilation outside of hospitals. In contrast, current guidelines recommend a follow-up care by the weaning centres that applies for patients with invasive ventilation at home. In an case the practical implementation of the follow-up care is not specified.

Material and Methods: In this paper we attempt to balance the reasons for the need of follow-up care for ventilated patients by the weaning centre on the basis of 6 cases. Furthermore, we want to report our experience with a weekly visitation of these patients as a basic structure of the followup care.

Results and Conclusions: The necessity for a regular consultation by a pneumologist that can be established by weekly rounds has been demonstrated. In addition, it can be expected to reduce the costs of public health markedly if one takes data from ventilated patients living in specialised institutions into account. Nevertheless prospective studies are necessary to objectify the financial and medical benefits arising from this form of medical supply. These studies should be designed as multicentre studies because of the heterogenous population of ventilated patients and the fact that home ventilation is in general a seldom occurrence. 


\section{Einleitung \\ $\nabla$}

In den letzten Jahren scheint die Häufigkeit von langzeitbeatmeten Patienten deutlich zugenommen zu haben. 2005 wurde nach einer Befragung von 483 Zentren die Prävalenz von Heimbeatmung in Europa auf 6,6/100000 Einwohner geschätzt, wobei es große regionale Unterschiede zwischen, aber auch innerhalb der einzelnen Länder gibt [1]. Von den heimbeatmeten Patienten mit pulmonaler Grunderkrankung sind etwa 10\% der Patienten tracheotomiert und invasiv beatmet, bei den throrakorestriktiven Erkrankungen waren etwa $5 \%$ und bei den neuromuskulären Grunderkrankungen etwa 20-25\% invasiv beatmet. Die Gründe für die vermeintliche Zunahme außerklinischer Beatmung liegt unter anderem an dem zusehends höheren Lebensalter der Patienten, einer Zunahme der Komorbiditäten sowie in einer $\mathrm{Zu}$ nahme der Anzahl chronisch Lungenkranker [2]. Auch die Zunahme von Expertise in der Heimbeatmung und die Zunahme an Beatmungszentren führt zu einer steigenden Prävalenz an Heimbeatmungspatienten [3].

Für die Entwöhnung von der invasiven Beatmung (Weaning) existiert mittlerweile eine gute Infrastruktur mit Zertifizierung der Weaningzentren über das „Weannet“. Je nach Patientenklientel ist dadurch in 50\%-70\% der Fälle ein erfolgreiches Weaning und Dekanülierung nach Langzeitbeatmung möglich. Etwa 25\% der Patienten versterben jedoch im Weaningzentrum und weitere $20 \%$ sind nicht von der invasiven Beatmung zu entwöhnen [4]. In einer Veröffentlichung wird in Italien innerhalb von 15 Jahren seit 1991 eine Abnahme des Weaningerfolgs von $87 \%$ auf $66 \%$ registriert, was in erster Linie auf zusehends schwerer erkrankte Patienten zurückzuführen sei [2].

Für diese Patienten mit außerklinischer invasiver Beatmung stehen derzeit keine flächendeckenden ärztlichen Versorgungsstrukturen zur Verfügung. Dabei wird sowohl in der Fachliteratur als auch in der aktuellen S2-Leitlinie „Nichtinvasive und invasive Beatmung als Therapie der chronischen respiratorischen Insuffizienz" für die Organisation der außerklinischen Beatmung eine poststationäre Mitbetreuung und eine Anbindung an das entlassende Weaningzentrum empfohlen [5]. Die Leitlinie formuliert dies imperativ: „Die außerklinische Beatmung muss um ein Weaningzentrum organisiert sein, der Arzt [des Weaningzentrums] ist für die Organisation der außerklinischen Versorgung verantwortlich." Die Art und Weise, wie diese Anbindung erfolgen und in der Praxis umgesetzt werden soll, bleibt dabei offen. Die Durchführungsempfehlung zur außerklinischen Beatmung, die vom Medizinischen Dienst des Spitzenverbandes Bund der Krankenkassen (MDS) und der AOK-Bundesverband (AOK-BV) mitverfasst wurde [6], sieht Hausbesuche als festen Bestandteil der Betreuung vor. Da der den Patienten betreuende Hausarzt in der Regel nicht über Erfahrungen auf dem Gebiet der außerklinischen Beatmung verfügt, wird die Empfehlung ausgesprochen, dass ein in der außerklinischen Beatmung erfahrener Arzt hinzugezogen wird.

Zur Zeit wird die Versorgung außerklinisch invasiv beatmeter Patienten im Wesentlichen von Pflegekräften spezialisierter Intensivpflegedienste und Hausärzten gewährleistet. Die ärztlichen Hausbesuche werden jedoch in der Regel nicht einmal wöchentlich durchgeführt, was teils an der geringen Vergütung, teils an dem großen Zeitaufwand liegen dürfte. Dies steht im augenfälligen Gegensatz zur Umgebung des Krankenhauses, wo der Patient zuvor auf der Intensivstation liegend in der Regel zweimal täglich visitiert wurde.
Im Rahmen des „Solinger Konzepts“ werden die Bewohner des Beatmungspflegeheims Haus Ahorn, das sich auf dem Betriebsgelände des Bethanien Krankenhauses Solingen in etwa 300 m Entfernung befindet, fachärztlich durch Pneumologen des Weaningzentrums betreut. Dies geschieht in Form einer wöchentlichen Visite, telefonischem Kontakt sowie in Form von Akutintervention im Fall von Notfällen. Diese Art der fachärztlichen Betreuung erfolgt dabei in enger Abstimmung mit dem Hausarzt, der unverändert die Basisversorgung übernimmt. Daten der Krankenkassen AOK Nordrhein/ Hamburg und TKK zeigen, dass hierdurch sowohl die Anzahl der stationären Wiederaufnahmen um 40\% als auch die Dauer der Krankenhausaufenthalte um 80\% reduziert werden. Die gemeinsame fach- und hausärztliche Versorgung führt dadurch zu einer immensen Kostenersparnis seitens der Kostenträger [8].

Bislang war diese Form der medizinischen Betreuung außerklinisch Beatmeter nur im Rahmen der stationären Pflege möglich. Es lag nahe, diese Versorgungsform auch für die außerklinische Beatmung derjenigen Patienten zu ermöglichen, die zu Hause gepflegt werden, und das bestehende Konzept um eine ambulante Betreuung zu erweitern. Dies ist durch einen Vertrag mit der AOK Rheinland/Hamburg möglich geworden, sodass hiervon zunächst nur Versicherte dieser Krankenkasse profitieren.

Das Grundkonstrukt der Nachsorge und Betreuung der Patienten ist dabei das gleiche wie bei stationärer Pflege: Bei Patienten, die im Weaningzentrum nicht entwöhnbar waren, werden nach Entlassung wöchentliche Hausbesuche durch einen pneumologischen Facharzt des Krankenhauses durchgeführt. Zusätzlich bestehen telefonische Kontakte zum Weaningzentrum und bei Notfällen besteht die Möglichkeit der Akutintervention vor Ort.

Dies geschieht in enger Abstimmung mit dem niedergelassenen Pneumologen, der eventuell ebenfalls in die Patientenversorgung involviert ist. Die Hausärzte übernehmen bei dieser Art der Betreuung weiterhin die Basisversorgung und geben ihr schriftliches Einverständnis zu dieser neuen Art der partnerschaftlichen Patientenbetreuung.

Da diese Art der Versorgung keine kassenärztliche Zulassung des Krankenhauses beinhaltet, werden weiterhin alle Verordnungen und Rezepte (Medikamente, Verbrauchsmaterial für die Beatmung etc.) durch den Hausarzt und den niedergelassenen Pneumologen ausgestellt. Dies stellt sicher, dass durch dieses Konzept keine Konkurrenz zwischen stationärem und ambulantem Sektor entsteht.

\section{Methodik und Ergebnisse}

Die Charakteristika von 6 Patienten in der häuslichen außerklinischen Beatmung sind beispielhaft in $\bullet$ Tab. 1 zusammengefasst. Funktion und Ablauf wöchentlicher Hausbesuche bei beatmeten Patienten ( $\bullet$ Tab. 2) lassen folgende Vorteile solch einer Versorgungsstruktur erkennen:

Der größte Vorteil besteht in der Behandlungskontinuität, sodass der Krankheitsverlauf des Patienten sehr gut bekannt ist. Idealerweise kennt der Arzt, der den Hausbesuch durchführt, den Patienten bereits persönlich von der Behandlung auf der Intensivstation.

Da die Prognose des Patienten in Kenntnis des Verlaufs und der Grunderkrankungen durch den Arzt des entlassenden Beatmungszentrums am besten eingeschätzt werden können, können hierdurch unnötige und unnötig lange stationäre Wiederaufnah- 
Tab. 1 Patientencharakteristika.

\begin{tabular}{|c|c|c|c|c|c|c|}
\hline Patient & 1 & 2 & 3 & 4 & 5 & 6 \\
\hline Alter & 83 Jahre & 75 Jahre & 63 Jahre & 62 Jahre & 66 Jahre & 71 Jahre \\
\hline Geschlecht & männlich & weiblich & weiblich & weiblich & weiblich & weiblich \\
\hline $\begin{array}{l}\text { beatmungsrelevante } \\
\text { Grunderkrankung }\end{array}$ & COPD & Bronchiektasen & $\begin{array}{l}\text { amyotrophe } \\
\text { Lateralsklerose }\end{array}$ & $\begin{array}{l}\text { amyotrophe } \\
\text { Lateralsklerose }\end{array}$ & $\begin{array}{l}\text { olivo-ponto-cere- } \\
\text { belläre Atrophie }\end{array}$ & $\begin{array}{l}\text { COPD, } \\
\text { Bronchiektasien }\end{array}$ \\
\hline Nebendiagnosen & $\begin{array}{l}\text { Epilepsie, } \\
\text { hypertensive } \\
\text { Herzerkrankung, } \\
\text { Vorhofflimmern, } \\
\text { Diab. mellitus, } \\
\text { sekundäre Neben- } \\
\text { niereninsuffizienz, } \\
\text { chronische Nieren- } \\
\text { insuffizienz }\end{array}$ & $\begin{array}{l}\text { Mamma-Karzinom, } \\
\text { arterielle Hypertonie, } \\
\text { Depression }\end{array}$ & $\begin{array}{l}\text { karnifizierende } \\
\text { Pneumonie, } \\
\text { hypertensive } \\
\text { Herzerkrankung }\end{array}$ & $\begin{array}{l}\text { Diabetes mellitus, } \\
\text { arterielle Hyper- } \\
\text { tonie }\end{array}$ & $\begin{array}{l}\text { Hashimoto- } \\
\text { Thryreoditis }\end{array}$ & $\begin{array}{l}\text { arterielle Hyper- } \\
\text { tonie, } \\
\text { koronare Herz- } \\
\text { erkrankung, } \\
\text { Z.n. NSTEMI }\end{array}$ \\
\hline $\begin{array}{l}\text { tägliche Beatmungs- } \\
\text { dauer }\end{array}$ & $24 \mathrm{~h}$ & $24 \mathrm{~h}$ & $24 \mathrm{~h}$ & $24 \mathrm{~h}$ & $24 \mathrm{~h}$ & $24 \mathrm{~h}$ \\
\hline $\begin{array}{l}\text { beatmet seit } \\
\text { (Wochen) }\end{array}$ & 18 & 51 & 67 & 52 & 164 & 15 \\
\hline $\begin{array}{l}\text { Intensivstation }{ }^{1} \\
\text { (Wochen) }\end{array}$ & 11 & 10 & 7 & 7 & 14 & 11 \\
\hline $\begin{array}{l}\text { außerklinisch } \\
\text { (Wochen) }\end{array}$ & 7 & 41 & 60 & 45 & 150 & 4 \\
\hline $\begin{array}{l}\text { Hausbesuche } \\
\text { (Wochen) }\end{array}$ & 7 & 41 & 55 & 42 & 35 & 2 \\
\hline Wiedereinweisungen ${ }^{2}$ & 0 & 0 & 4 & 2 & 2 & 1 \\
\hline
\end{tabular}

${ }^{1}$ Intensivstation = Dauer der Behandlung auf Intensivstation (erstversorgende Krankenhäuser und Weaningzentrum zusammengerechnet) vor der ersten Entlassung nach Hause

2 Wiedereinweisungen = nach Beginn der Hausbesuche durch das Weaningzentrum

Tab. 2 Funktion und Ablauf der Hausbesuche.

- Herstellen einer Behandlungskontinuität
- Initiierung von Therapielimitierung und Therapierückzug
- Vermitteln von Krankheitslehre
- Durchführung von Kanülenwechsel
- Wundversorgung von Tracheotomien (z. B. Granulationen,
Entzündungen)
- Organisation von (Beatmungs-) Hilfsmitteln
- Anpassen von Beatmungsintensität und der Höhe der Sauerstoffgabe
- Durchführung von Sekretolyse
- Anpassen der oralen oder Sondenkost, Erfassen von Schluckstörungen
- Kommunikation mit Akutkrankenhäusern und Organisation nicht-
beatmungsassoziierter Krankenhausaufnahmen
- Qualitätskontrolle von Beatmungszentrum und Pflegedienst
- Überprüfung der Indikation für die Fortsetzung der Beatmung
- Ausbau von Spontanatmungsphasen
- Evaluation der Möglichkeit der späten Beatmungsentwöhnung
- Moderation von Rückzugspflege (partielle Übernahme der Pflege-
tätigkeit durch Familienmitglieder)
- Moderation von Palliativsituationen

men vermieden werden, was hilft, die Kosten in der außerklinischen Beatmung zu reduzieren.

In diesem Zusammenhang ist das Thema der Therapiebegrenzung von Bedeutung. Gespräche mit Patienten und Angehörigen über Therapiebegrenzung oder Therapierückzug werden häufig im Weaningzentrum initiiert. Nach der Entlassung sind die Ergebnisse solcher Entscheidungen häufig nicht mehr bekannt oder aber die Umsetzung fällt schwer, weil der Patient dem Pflegedienst oder dem Hausarzt nicht bekannt ist und der intensivmedizinische Verlauf der letzten Wochen nicht miterlebt wurde, sodass eine prognostische Einschätzung erschwert ist. Teilweise geben auch Notfallpläne der Pflegedienste vor, dass im Fall einer akuten Verschlechterung des Patienten pauschal der Notarzt zu verständigen sei.
Durch die Hausbesuche kann sowohl die Kontinuität einer Palliativbehandlung gewährleistet als auch eine Palliativtherapie neu initiiert und moderiert werden.

Die Krankheitsbilder der zur Beatmung führenden Erkrankungen und die sich daraus ergebenden Probleme sind den vor Ort tätigen Pflegekräften und auch den Hausärzten nicht immer bekannt. Wenn kein niedergelassener Facharzt (Pneumologe) in die Behandlung durch Hausbesuche integriert ist, kommt dem betreuenden Arzt des Weaningzentrums die Rolle des Weiterbilders zu. Spezielle Probleme (z.B. fehlender Hustenstoß und Sekretverhalt bei neuromuskulären Patienten) können dadurch gezielt angesprochen und Therapieversuche unternommen werden.

Der wöchentliche Hausbesuch wird außerdem dazu genutzt, den je nach Kanülentyp alle 2 bis 4 Wochen notwendigen Kanülenwechsel gemeinsam mit der Pflegekraft durchzuführen, was die Sicherheit für diese Prozedur insgesamt erhöht. Dadurch ist es auch möglich, die Pflegekräfte vor Ort im Kanülenwechsel zu schulen. Des Weiteren können so Probleme mit dem Tracheostoma früher erfasst und geeignete Maßnahmen getroffen werden (z.B. Therapie von Wundgranulationen oder Entzündungen).

Auch Veränderungen in den Respiratoreinstellungen können sinnvoll nur vor Ort im Rahmen eines Hausbesuches erfasst werden. Dadurch erhalten auch die Weaningzentren Rückmeldung, ob die Beatmungseinstellungen, mit denen die Patienten entlassen werden, tatsächlich für außerklinische Beatmung geeignet sind.

Im weiteren Krankheitsverlauf können mit Änderung des Allgemeinzustands des Patienten jederzeit Veränderung der Beatmungsintensität oder -dauer notwendig werden. Dies festzustellen und festzulegen ist ebenfalls Aufgabe des Pneumologen [6].

Bei Bedarf werden sekretolytische Maßnahmen, insbesondere die Therapie mit beispielsweise dem Cough Assist, durch den Arzt vor Ort durchgeführt und das Gerät entsprechend der Be- 
dürfnisse des Patienten eingestellt. Zuweilen ist das Heranführen des Patienten an technische Hilfsmittel schwierig und erfordert einige Geduld.

Ein weiterer Schwerpunkt bei den Hausbesuchen bildet die Ernährung. Die Ernährung bei Beatmungspatienten ist ein Schwerpunkt der Intensiv- und Beatmungsmedizin und nicht hausärztlich verankert. Dabei wird während der Hausbesuche regelmäßig die Art und der Umfang von Sondenkost genauso wie das Ausmaß des oralen Kostaufbaus diskutiert. Schluckstörungen müssen zuverlässig erfasst und gegebenfalls einer logopädischen Therapie zugeführt werden.

Die gute Vernetzung der Weaning- und Beatmungszentren mit anderen Krankenhäusern ist hilfreich bei der Organisation von stationären Aufnahmen, die wegen nicht-beatmungsassoziierter Erkrankungen erfolgen müssen. Beispielweise könnten Gallenkoliken ein Grund für eine stationäre Behandlung darstellen. Normalerweise würde dies eine stationäre Aufnahme auf Normalstation bedeuten. Die Beatmungspflichtigkeit des Patienten führt jedoch dazu, dass in dieser Situation eine Aufnahme auf der Intensivstation notwendig wird. Die Intensivkapazitäten von Akutkrankenhäusern sind jedoch begrenzt und daher ist es oft schwierig, diese Patienten an ein geeignetes Krankenhaus zu vermitteln. Wir sehen daher die Organisation von stationären Aufnahmen als Aufgabe des betreuenden Facharztes. Tab. 4 fasst die Gründe für stationäre Wiederaufnahmen in dem betreuten Patientenkollektiv zusammen.

Die Dauer der täglich notwendigen Behandlungspflege (Beatmungspflege) wird durch den behandelnden Arzt festgelegt. In den Fällen, in denen Angehörige Behandlungspflege übernehmen sollen, besteht häufig eine Konfliktsituation, in der sich die Angehörigen mit der Übernahme von Pflegetätigkeit unsicher und überfordert fühlen. Fehlt in dieser Situation die fachärztliche Moderation, sind langwierige Konflikte zwischen Kostenträgern, Intensivpflegedienst und Familie des Patienten vorprogrammiert.

Die Indikation für die Fortführung der Beatmung und die Reevaluation der Beatmungsparameter wird von manchen Zentren durch eine stationäre Wiederaufnahme überprüft. Dieses Vorgehen wird sowohl in der Leitlinie als auch in der Durchführungsempfehlung zur außerklinischen Beatmung empfohlen [5, 6].

Im Rahmen eines Hausbesuchs kann jedoch auch die Möglichkeit einer späten Beatmungsentwöhnung evaluiert werden. Soweit zumindest ein Pulsoxymeter vorhanden ist, kann eine sukzessive Ausdehnung der Spontanatmungsphasen versucht werden, was dem Patienten zu größerer Autonomie verhilft und den Umfang der Behandlungspflege reduziert. Sollte sich abzeichnen, dass eine vollständige Respiratorentwöhnung möglich wäre, fände eine Rückverlegung ins Zentrum zur Komplettierung des Weanings statt.

\section{Diskussion}

Wir sehen eine Notwendigkeit zur fachärztlich-pneumologischen Betreuung ( $\bullet$ Tab.3), die sich zum einen aus der Komplexität der Beatmungssituation ergibt und zum anderen aus der Tatsache, dass es häufig pneumologische Erkrankungen sind, die zur dauerhaften Beatmung führen.

Da auch neurologische Krankheitsbilder, die mit einer Atrophie der Atempumpe und einer chronischen ventilatorischen Insuffizienz einhergehen, regelmäßig von Pneumologen in Weaningzentren betreut werden, existiert dort eine besondere Expertise
Tab.3 Vorteile der Nachsorge durch das entlassende Beatmungszentrum.

- poststationäre Mitbetreuung und eine Anbindung an das entlassende Beatmungszentrum, empfohlen in der S2-Leitlinie „Nichtinvasive und invasive Beatmung als Therapie der chronischen respiratorischen Insuffizienz"

- keine fachärztliche Anbindung trotz gravierender, teils seltener Krankheitsbilder

auch durch Hausarzt oft nur selten Hausbesuche

- Vermeidung von unnötigen oder unnötig langen stationären

Aufenthalten

Tab.4 Gründe für stationäre Wiedereinweisungen der Patienten (in Klammern Dauer der stationären Behandlung).

\begin{tabular}{|c|c|}
\hline $\begin{array}{l}\text { Patient } 3 \\
\text { amyotrophe } \\
\text { Lateralsklerose }\end{array}$ & $\begin{array}{l}\text { 1. Pneumonie (6 Tage) } \\
\text { 2. Hypoxie bei Sekretverlegung, } \\
\text { Pneumonie ( } 14 \text { Tage) } \\
\text { 3. hohes Fieber ohne Fokusnachweis } \\
\text { (6 Tage) } \\
\text { 4. pseudomembranöse Kolitis (4 Tage) } \\
\text { 5. pneumogene, katecholaminpflichtige } \\
\text { Sepsis ( } 16 \text { Tage) } \\
\text { 6. Sturz mit Beckenprellung ( } 2 \text { Tage) } \\
\text { 7. Diarrhoen, Pneumonie, Harnwegsinfekt } \\
\text { (12 Tage) } \\
\text { 8. Obstipation ( } 1 \text { Tag) } \\
\text { 9. Fieber, Diarrhoe, transfusionspflichtige } \\
\text { Anämie (6 Tage) }\end{array}$ \\
\hline $\begin{array}{l}\text { Patient } 4 \\
\text { amyotrophe } \\
\text { Lateralsklerose }\end{array}$ & $\begin{array}{l}\text { 1. schwieriger Trachealkanülenwechsel } \\
\text { ( } 1 \text { Tag) } \\
\text { 2. hypertensive Entgleisung mit } \\
\text { Lungenödem (8 Tage) }\end{array}$ \\
\hline $\begin{array}{l}\text { Patient } 5 \\
\text { olivo-ponto-cerebelläre } \\
\text { Atrophie }\end{array}$ & $\begin{array}{l}\text { 1. Verschlechterung des Allgemein- } \\
\text { zustandes, Diarrhoen (14 Tage) }\end{array}$ \\
\hline $\begin{array}{l}\text { Patient } 6 \\
\text { COPD, Bronchiektasen }\end{array}$ & $\begin{array}{l}\text { 1. pneumogene Sepsis, verstorben } \\
\text { (6 Tage) }\end{array}$ \\
\hline
\end{tabular}

für die Atmungsinsuffizienz bei seltenen Erkrankungen (z.B. amyotrophe Lateralsklerose, Muskeldystrophien, M. Gaucher, mitochondrialen Myopathien usw.). Daher sind sowohl die fachärztlich-pneumologische Anbindung im allgemeinen als auch die Anbindung an ein Weaningzentrum im Speziellen sinnvoll.

Der wöchentliche Hausbesuch stellt zu allervorderst eine vertrauensbildende Maßnahme für den Patienten und dessen Angehörigen dar, die sich dadurch besser in die Situation einfinden können. Auch persönliche Konflikte zwischen Pflegekräften und Angehörigen und/ oder Betroffenen können von dieser Position des Vertrauens aus durch den Arzt moderiert werden.

Für das Weaningzentrum bietet die Nachbetreuung der Patienten in Form von Hausbesuchen die Möglichkeit, den tatsächlichen weiteren klinischen Verlauf zu erfassen. Ein markantes Merkmal der Qualität der außerklinischen Beatmung ist dabei die Häufigkeit von stationären Wiedereinweisungen. Durch die Hausbesuche ist sowohl eine Qualitätskontrolle gegenüber dem Pflegedienst als auch gegenüber der eigenen Arbeit möglich.

Für die Pflegedienste bedeutet die Anbindung an den Arzt des Weaningzentrums, dass die Einzelbetreuung des Patienten aufgebrochen und stattdessen analog zur stationären Intensivtherapie ein gemeinsames ärztlich-pflegerisches Versorgungsteam gegründet wird. Der Arzt steht dabei zusätzlich zu den Hausbesuchen im engen telefonischen Kontakt mit der Pflegekraft vor Ort. Trotz alledem erfolgt die Betreuung der Beatmungspatienten in enger Abstimmung mit dem Hausarzt, dessen Zustimmung zu 
dieser Kooperation erforderlich ist. Die hausärztliche Betreuung ergänzt die fachärztliche Nachsorge nicht nur, unter Umständen können in der außerklinischen Beatmungssituation durchaus hausärztliche Belange im Vordergrund stehen (z.B. Einstellung eines erhöhten Blutzuckers). Ferner erfolgt die Verordnung von Medikamenten und Therapien allein durch den Hausarzt, da die Beatmungszentren nicht über die entsprechende ambulante Kassenzulassung verfügen.

Problematisch bleibt, dass die Vergütungsfrage für die Beatmungszentren nicht geklärt ist. Es kann versucht werden, über Honorarverträge mit Pflegediensten oder Einzelverträge mit Krankenversicherern ein Entgelt zu sichern. Die vermeintlichen Mehrkosten rechnen sich für die Versicherer, da die hohen Kosten für die außerklinische Beatmung nicht zuletzt von der Dauer und Häufigkeit der stationären Wiederaufnahmen bestimmt werden und erwartet werden darf, dass diese durch die Anbindung an das Weaningzentrum deutlich reduziert werden. Dies konnte für die stationäre Beatmungspflege bereits belegt werden.

Die Bereitschaft der Weaningzentren, in Vorleistung zu gehen und die poststationäre Nachsorge in Form von Hausbesuchen zunächst unentgeltlich anzubieten, ist gering. Dies führt jedoch da$\mathrm{zu}$, dass die Weaningzentren keine Expertise in der Betreuung außerklinisch beatmeter Patienten entwickeln und der strukturelle Bedarf gegenüber den Kostenträgern nicht plausibel gemacht werden kann. Letztlich wird dadurch suggeriert, dass es keine Notwendigkeit für die fachärztliche Versorgung durch den in Beatmungsmedizin erfahrenen Pneumologen gibt.

\section{Schlussfolgerung}

$\nabla$

Wir sehen gute Argumente für die Nachsorge der außerklinisch Beatmeten durch das entlassende Weaningzentrum. Wir halten dazu wöchentliche Hausbesuche für geeignet, die sich schnell etablieren lassen und zum festen Bestandteil der außerklinischen
Beatmung werden können. Unsere Erfahrungen mit den Hausbesuchen sind überaus positiv. Es fehlen dennoch prospektive Studien, um den finanziellen und medizinischen Vorteil durch diese Versorgungsstruktur objektiv einschätzen zu können. Da die außerklinische Beatmung insgesamt selten ist, werden solche Studien multizentrisch ausgelegt sein müssen. Werden die Daten aus der Betreuung von Heimbewohnern zugrunde gelegt, ist neben einer Verbesserung der Lebensqualität und von einer deutlichen Kostenersparnis des Gesundheitswesens auszugehen.

\section{Interessenkonflikt}

$\nabla$

Die Autoren geben an, dass kein Interessenkonflikt besteht.

\section{Literatur}

1 Lyloyd-Owen SJ, Donaldson GC, Ambrosino N et al. Patterns of home mechanical ventilation use in Europe: results from the Eurovent survey. Eur Respir J 2005; 25: 1025 - 1031

2 Polverino E, Nava S, Ferrer M et al. Patients' characterization, hospital course and clinical outcomes in five Italian respiratory intensive care units. Intensive Care Med 2010; 36: 137-142

3 Dybwik $K$, Tollali T, Nielsen EW et al. Why does the provision of home mechanical ventilation vary so widely. Chronic Respiratory Disease 2010; 7: 67-73

4 Scheinhorn DJ, Hassenpflug MG, Votto JJ et al. Post-ICU Mechanical Ventilation at 23 Long-term Care Hospitals. Chest 2007; 131: 85-93

5 Windisch $W$ et al. Nichtinvasive und invasive Beatmung als Therapie der chronischen Insuffizienz. Pneumologie 2010; 64: 207-240

6 Randerath WJ, Kamps N, Brambring J et al. Durchführungsempfehlungen zur außerklinischen invasiven Beatmung. Pneumologie 2011; 65: $72-88$

7 Schönhofer B, Pfeifer M. Weannet - Kompetenznetzwerk pneumologischer Weaningzentren. Der Pneumologe 2010; 7: 121-124

8 Randerath $W$. Integrative medizinische und pflegerische Versorgung langzeitbeatmeter Patienten. In: Randerath W Hrsg. Praktische Aspekte der Heimbeatmung. Bremen: Uni-med; 2010; 1: Aufl. 58-62

9 Otterbein J. Überleitmanagement: Nahtstelle zwischen klinischer und außerklinischer Beatmung. In: Randerath W Hrsg. Praktische Aspekte der Heimbeatmung. Bremen: Uni-med; 2010; 1: Aufl. 58-62 